\title{
Fischhäckselanlagen und Vogelschreddermaschinen
}

\author{
Was ist ein Windrad? Eine Wunderwaffe im Klimaschutz, sagen die einen. \\ Ein ökologisches Verbrechen, sagen die anderen. Der Ausbau erneverbarer \\ Energien ist ein Konfliktfall zwischen Umwelt- und Klimaschützern geworden. \\ Erste Lösungswege zeichnen sich bereits ab, doch der Einsicht muss mancherorts \\ noch auf die Sprünge geholfen werden.
}

$\mathrm{N}$ Von Frank Musiol icht erst sei dem umstrittenen Windenergie-Verriss des Spiegels im Frühjahr 2004 ist bekannt, dass der Ausbau der Windenergie in Deutschland voller Konflikte ist. Es streiten aber nicht nur die Vertreter einer um ihre Wettbewerbsfähigkeit besorgten deutschen Großindustrie mit den Vertretern einer das Klima schonende Energieversorgung. Auch bei Bürgerinnen und Bürgern stehen sich Befürworter und Kritiker immer öfter gegenüber. Umfragen bestätigen zwar, dass der überwiegende Teil der Deutschen den Ausbau der Erneuerbaren Energien begrüßt. Doch vor Ort regt sich immer öfter zum Teil organisierter Widerstand. Und nicht selten mischen Aktive der großen Umweltverbände kräftig mit. Ein Zeichen dafür, dass es auch innerhalb der Umweltszene Konflikte gibt. Das sollte nicht verwundern, wenn man sich vor Augen führt, wie weit die Skala der im Umweltschutz Aktiven reicht. Kümmern sich die einen um globale Umweltprobleme, liegt den anderen der Schutz lokaler Natur am Herzen. Weder dem einen noch dem anderen sollte man im übrigen abstreiten, hehre Ziele zu verfolgen.

Zwar ist der Streit um die Windenergie am stärksten in der Öffentlichkeit präsent, doch beim Grundkonflikt um die Nutzung Erneuerbarer Energien geht es um mehr. Denn auch bei allen anderen Formen Erneuerbarer Energien - abgesehen vielleicht von der Geothermie - treten Nutzungskonflikte auf: Kleinwasserkraftwerke sind ein Eingriff in die Fließgewässerökologie, der Anbau von Energiepflanzen birgt die Gefahr der Ausbreitung intensiv bewirtschafteter Monokulturen und Photovoltaik drängt zunehmend von Dächern in die Fläche, so dass auch hier Flächenverbrauch und Landschaftsbeeinträchtigung zum Problem werden können.

\section{- Konfliktlöser EEG}

Mit den Konflikten zwischen Klima- und Naturschutz mochten sich auch viele Politiker der Regierungskoalition in der Vergangenheit nicht gerne auseinander setzen. Die Erfolgsstory der Erneuerbaren Energien war ihnen geradezu heilig. Doch wer diesen Erfolg langfristig fortsetzen will, muss versuchen, Konflikten mit anderen, ebenso wichtigen Facetten des Umweltschutzes zukünftig gar nicht erst entstehen zu lassen. Vor diesem Hintergrund gab es in den vergangenen anderthalb Jahren eine ausführliche Debatte über eine Verankerung von Naturschutzaspekten in der Novelle des Erneuerbare Energien Gesetzes (EEG). Für viele war die Gemengelage in dieser Diskussion ziemlich undurchschaubar. Da engagierten sich Umweltverbände zum einen für eine Aufstockung der Vergütungen in allen Bereichen, auf der anderen Seite aber auch dafür, dass Anlagen an nicht naturgerechten Standorten aus der Vergütung herausgenommen werden. Einen erbitterten Streit hat es in diesem Zusammenhang über die kleine Wasserkraft gegeben. Deren Ausbau gilt in weiten Teilen der Umweltszene seit Jahren als Unsinn. Die Potenziale für unsere Stromversorgung sind aufgrund der kleinen Anlagengrößen unbedeutend, dafür ist der ökologische Schaden, wenn Anlagen an bisher unverbauten Gewässerabschnitten errichtet werden, umso größer.

Mit dem Kompromiss, der schließlich gefunden wurde, sollten eigentlich alle leben können. Von den Umweltverbänden wurde er jedenfalls durchweg begrüßt. Die Vergütungssätze sind insgesamt den wirtschaftlichen Erfordernissen angepasst worden und es sollte nun gewährleistet sein, dass nach der Windenergie auch die anderen Erneuerbaren Energien - allen voran die Biomasse - einen kräftigen Aufschwung er- leben. Daneben sind einige Restriktionen im EEG formuliert, die den Streit um Naturschutzaspekte zukünftig entschärfen sollten.

Ab 2008 sollen neue kleine Wasserkraftanlagen mit einer Leistung von weniger als fünfhundert Kilowatt nur noch dann in die Vergütung kommen, wenn sie im Zusammenhang mit ganz oder teilweise bestehenden Querverbauungen errichtet werden und ein guter ökologischer Zustand des Fließgewässers erreicht beziehungsweise der ökologische Zustand wesentlich verbessert wird. Das ist nicht mehr und nicht weniger als die Europäische Wasserrahmenrichtlinie ohnehin vorschreibt. Dafür gibt es aber eine um zwei Cent höhere Vergütung, womit sich auch die Modernisierung älterer Anlagen mit ökologischen Begleitmaßnahmen rechnen sollte. Doch die Wasserkraftlobby kämpft bis heute verbissen gegen das Einverständnis an, dass Wasserkraftnutzung die Fließgewässerökologie nun einmal negativ beeinflussen kann. Diese Kompromissunfähigkeit müsste man tatsächlich anprangern, gäbe es nicht auf Seiten der Naturschützer auch einzelne Stimmen, die im Prinzip den Rückbau der gesamten Wasserkraft anstreben.

\section{- Neverungen}

Weitere Regelungen, die aus Gründen des Naturschutzes in die Novelle des EEG aufgenommen wurden, sind:

- Offshore-Windparks, die ab 2005 genehmigt werden, kommen nur dann in die Vergütung, wenn sie nicht in Natura-2000-Gebieten liegen. Damit wird eine wichtige Forderung des NABU zur Entwicklung der Offshore-Windenergie erfïllt. Im Gegenzug wird die Anschlussfrist für die Maximalvergütung um vier Jahre verlängert und die Vergütung steigt mit zunehmender Küstenentfernung und Wassertiefe an.

- Strom aus Biomasse wird um vier bis sechs Cent höher vergütet, wenn nur Pflanzenbestandteile und keine sonstigen Reststoffe verstromt werden.

- Photovoltaikanlagen auf Freiflächen fallen nur unter die Vergütung, wenn sie auf bereits vorbelasteten Flächen oder innerhalb eines gültigen Bebauungsplans errichtet werden. Wird der Bebauungsplan für eine solche Anlage extra erweitert, muss es sich um Ackerland handeln, das gleichzeitig in Grünland umgewidmet wird.

Problematisch an dem aktuellen Streit ist der Eindruck, Naturbeeinträchtigungen durch Energienutzung würden erst seit der Ausbreitung der Erneuerbaren Energien richtig thematisiert wer- 
den. Dadurch entsteht allerdings ein schiefes Bild. Durch die Nutzung fossiler und nuklearer Energieträger wurden und werden Gleichgewichte der Natur auf völlig unakzeptable, dramatische Weise ge- oder zerstört, Mensch und Natur der Landschaft beraubt und langfristigen Schädigungen ausgesetzt. Dessen ist sich die Umweltbewegung seit jeher bewusst und deshalb gehört die Energiewende seit vielen Jahren zu ihren Kernanliegen. Daran hat sich im Grundsatz nichts geändert, es ist lediglich die Erkenntnis hinzugekommen, dass jede Energienutzungsform mit Eingriffen in den Naturhaushalt verbunden ist. Die sind zwar - zumindest global betrachtet - bei den Erneuerbaren deutlich geringer, trotzdem muss eine Diskussion darüber erlaubt sein, wie auch diese Beeinträchtigungen soweit wie möglich vermieden werden können. Im Prinzip sollte auch hier das Minimierungsgebot greifen, auf das wir uns so oft berufen.

Dass Proteste gegen die Windenergie scheinbar umfangreicher werden als solche gegen Kohle und Atom ist auch eine Begleiterscheinung der Dezentralisierung unserer Energieversorgung. Von ein paar tausend Windrädern fühlen sich nun einmal mehr Menschen direkt betroffen als von einem Braunkohletagebau oder einem Atomkraftwerk. Der vermeintlichen Landschaftsverschandelung durch einzelne Windparks die drastische Landschaftszerstörung im Braunkohlerevier entgegen zu halten, nützt da wenig. Deshalb muss man sich mit der Kritik detailliert auseinandersetzen. Sachliche Aufklärungsarbeit kann viele Ängste - zum Beispiel vor Lärmbelastung oder Schattenwurf - ausräumen oder zumindest relativieren. Prinzipielle Ablehnung, aus welchen Gründen auch immer, ändert man damit nicht. Einen gutes Stiick weit trifft nämlich auf viele Windkraftgegner das zu, was Atomkraftgegnern früher häufig vorgeworfen wurde: sie sind ideologisch motiviert. Anders lässt sich die zum Teil hanebüchene und platte Argumentation - zum Beispiel aus den Reihen des so genannten Bundesverbands Landschaftsschutz - nicht erklären.

\section{- Protest ist nicht gleich Protest}

Mit dieser Art von Protest dürfen sich die Umweltverbände nicht in einen Topf werfen lassen. Wollen sie ihre Glaubwürdigkeit nicht verlieren, müssen sie sich von Polemik distanzieren und stattdessen sachliche Kritik gegen konkrete Erneuerbare-Energie-Anlagen herausarbeiten. Wo es solche nicht gibt, gibt es gute Gründe, Projekte auch vor Ort zu unterstiutzen. Vielfach ist dafür innerhalb der Umwelt- und Naturschutzverbände aber noch Aufklärungsarbeit gefragt. Bekenntnisse der Bundesverbände zu Klimaschutz und Erneuerbaren Energien nützen in der Praxis wenig, wenn Ortsgruppen davon unbeeindruckt gegen Windräder zu Felde ziehen. Auch das gibt es. Dass den Ehrenamtlichen vor Ort die Vogelwelt in ihrer Umgebung zunächst einmal wichtiger ist als die Klimaschutzstrategie der Bundesregierung, ist verständlich. Dass aber ein langfristig erfolgreicher Naturschutz auch von stabilen klimatischen Bedingungen abhängt, ist bisher häufig ungenügend vermittelt.

Ein „Entweder-Oder“ kann es trotzdem nicht geben. Vielmehr muss nach Wegen gesucht werden, wie man beide Ziele überein bringen kann. Vielfach wird in diesem Zusammenhang auf die Entwicklung von Standards gesetzt. Regeln für Planer also, die bei Einhaltung dazu führen sollen, dass eine Beeinträchtigung von Mensch und Natur nicht zu befürchten ist. Solche Standards sind zu begrüßen. Lokale Konflikte werden sie aber dennoch in der Regel nicht vermeiden können. Das Zauberwort dürfte vielmehr in vielen Fällen „Kompromiss“ heißen. Kann ein umstrittener Windpark nicht an einem anderen Standort errichtet werden? Kann vielleicht die Anlagenzahl verringert werden? Kann eine Wasserkraftanlage durch zusätzliche Maßnahmen naturverträglicher werden oder sich eine Photovoltaikanlage besser ins Landschaftsbild einfügen?

\section{> Strategien zur Konfliktlösung}

Um sich über solche Fragen einer Konfliktlösung nähern zu können, muss man miteinander reden und schon daran scheitert es in der Praxis häufig. Planer halten es oft für mühsam und Behörden für überflüssig, die Naturschutzverbände an der Planung frühzeitig zu beteiligen - sie könnten ja auf Ablehnung stoßen. Andererseits kommt es auch vor, dass örtliche Naturschützer sich Gesprächen verschließen und lieber regionalen Protestbewegungen anschließen. Beides keine guten Voraussetzungen, in der Sache voran zu kommen.

Diesen Stillstand will der Naturschutzbund (NABU) überwinden. Als ersten Schritt haben wir im Mai dieses Jahres einen Workshop veranstaltet, auf dem die einzelnen Akteure über Perspektiven der Konfliktvermeidung diskutierten (1). In einem Projekt, das wir in den kommenden anderthalb Jahren mit Unterstützung des Bundesumweltministeriums und Umweltbundesamts durchführen, widmen wir uns nun intensiv der naturschutzfachlichen Auseinandersetzung um den
Ausbau der Erneuerbaren Energien und insbesondere örtlich auftretenden Konfliktfällen (2). Als eine Art Clearingstelle, die für alle Beteiligten ansprechbar ist, versucht ein kleines Team in der NABU-Bundesgeschäftsstelle in solchen Fällen zu vermitteln. In der Regel wird das wichtigste sein, die vermeintlichen Kontrahenten an einen Tisch zu bringen. Dabei geht es nicht darum, Ortsgruppen ,auf Linie“ zu bringen oder sie gar zu bevormunden. Vielmehr werden wir dort Hilfestellung anbieten, wo Ehrenamtliche vor Ort mit Planungen überfordert sind. Wo sie jedoch argumentativ auf dem Holzweg sind, werden wir versuchen, Überzeugungsarbeit zu leisten. Überzeugungsarbeit leisten wir aber auch dort, wo Naturschutzargumente geflissentlich übergangen werden. Denn auch die Erneuerbaren-Branche muss einsehen, dass wir nur miteinander am Ziel ankommen.

Wir werden nicht überall vermitteln und zu Kompromissen finden können. Auch organisierten Protest werden wir nur selten aufhalten. Dazu wird es in vielen Fällen schlichtweg zu spät sein. Wir haben aber nicht nur die Möglichkeit, die eine oder andere Einigung zu erzielen. Wir haben auch die Chance, aus Erfahrungen mit Konflikten und ihren Entstehungsgründen zu lernen und daraus Strategien für zukünftige Konfliktvermeidung zu entwickeln.

\section{Anmerkungen}

(1) NABU ( $\mathrm{Hg}$. ): Naturschutz kontra Erneuerbare Energien? Konfliktlösungsstrategien für die Praxis. NABU-Tagung, Bonn, 19. Mai 2004. Tagungsband beim Autoren erhältlich. (2) NABU-Projekt „Konfliktlösungsstrategien Erneuerbare Energien", Laufzeit 6/04 bis 3/06.

\section{Der Autor}

Dr. Frank Musiol ist Referent für Klima und Energie beim Naturschutzbund (NABU) Bundesverband. Kontakt: NABU, Herbert-Rabius-Straße 26, 53225 Bonn. Tel. 0228-4036164, E-Mail: Frank.Musio|@NABU.de 
(c) 20I0 Authors; licensee IÖW and oekom verlag. This is an article distributed under the terms of the Creative Commons Attribution Non-Commercial No Derivates License (http://creativecommons.org/licenses/by-nc-nd/3.o/), which permits unrestricted use, distribution, and reproduction in any medium, provided the original work is properly cited. 